\title{
Método sol-gel modificado para obtenção de alumina nanoencapsulada com terras raras
}

\section{(Sol-gel modified method for obtaining $\alpha$-alumina nanocoated with rare earth)}

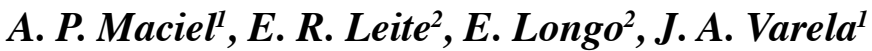 \\ ${ }^{1}$ Instituto de Química, UNESP, Araraquara, SP 14801-900 \\ ${ }^{2}$ CMDMC, LIEC, Departamento de Química, UFSCar, \\ Via Washington Luiz, km 235, S. Carlos, SP 13565-905 \\ adeilton@liec.ufscar.br
}

\begin{abstract}
Resumo
Neste trabalho, $\alpha$-alumina foi nanoencapsulada com óxido de lantânio usando um método sol-gel modificado. Um precursor polímero de lantânio foi usado para encapsular a $\alpha-\mathrm{Al}_{2} \mathrm{O}_{3}$, dispersa em água. A influência da nanocápsula de óxido de lantânio na sinterização e microestrutura da $\alpha$-alumina foi investigada usando-se técnicas de microscopia eletrônica. A microscopia eletrônica de varredura dos grãos nanoencapsulados revelou que compostos de lantânio precipitam no contorno de grão da $\alpha$ - $\mathrm{Al}_{2} \mathrm{O}_{3}$. $\mathrm{A}$ microscopia eletrônica de alta resolução mostrou claramente a formação de uma camada de $\mathrm{La}_{2} \mathrm{O}_{3}$ na superfície da alumina. A camada de $\mathrm{La}_{2} \mathrm{O}_{3}$ modifica o processo de sinterização da alumina pura. Em temperaturas elevadas ocorrem mudanças significativas na morfologia e microestrutura da $\alpha$-alumina. Verificou-se que a camada amorfa de $\mathrm{La}_{2} \mathrm{O}_{3}$ reage com a alumina, resultando em $\mathrm{LaAlO}_{3}$, aumentando a temperatura de sinterização. A fase $\mathrm{La}_{2} \mathrm{Al}_{24,4} \mathrm{O}_{39,6}$ foi observada no material sinterizado.
\end{abstract}

Palavras-chave: $\alpha-\mathrm{Al}_{2} \mathrm{O}_{3}$, nanocápsula, terra-rara.

\begin{abstract}
$\alpha$-alumina powder was nanocoated with lanthanum oxide by sol-gel like method. A lanthanum polymeric precursor was used to coat the $\alpha-\mathrm{Al}_{2} \mathrm{O}_{3}$ dispersed in water. The influence of the lanthanum oxide nanocoating on the sintering and microstructure of $\alpha$-alumina was investigated using electron microscopy techniques. Scanning electron microscopy (SEM) of these nanocoated grains revealed that the lanthanum oxide precipitates on the grain boundary of the $\alpha$-alumina, at high temperature. High-resolution electron microscopy (HREM) observations clearly showed the formation of a $\mathrm{La}_{2} \mathrm{O}_{3}$ layer on the surface of the $\alpha$-alumina. The La $\mathrm{O}_{3}$ layer modified the sintering process of the pure alumina. At high temperatures significant modifications on the grain morphology and microstructure was observed. It was verified that an amorphous $\mathrm{La}_{2} \mathrm{O}_{3}$ layer reacts with the $\alpha$-alumina grain, resulting in LaAlO and increasing the sintering temperature. The $\mathrm{La}_{2} \mathrm{Al}_{24,4} \mathrm{O}_{39,6}$ phase was observed.
\end{abstract}

Keywords: $\alpha-\mathrm{Al}_{2} \mathrm{O}_{3}$, nanocoating, rare-earth.

\section{INTRODUÇÃO}

O processo de sinterização pode ser facilitado por fatores geométricos e pela alta viscosidade do material, como ocorre na sinterização de pequenas esferas de vidro. Este comportamento, no entanto, não é observado em pós cerâmicos policristalinos, uma vez que estes materiais apresentam anisotropia nas energias das superfícies. Assim, existe uma maior complexidade para se entender a sinterização nesse último caso. Este processo é um fenômeno termicamente ativado e controlado por difusão. Então, o transporte de massa figura-se como um fator importante no entendimento das características deste fenômeno e a presença de impurezas, dopantes ou aditivos deve ser considerada [1].
A sinterização de pós cerâmicos policristalinos ainda está sujeita a competição de outros processos que ocorrem com o aumento da temperatura. Um dos principais concorrentes é o crescimento de grão, que depende sensivelmente da difusão superficial do material [2].

A difusão superficial e a ativação de difusão do contorno de grão podem ser controladas por meio de pequenas quantidades de aditivos. Como os mecanismos de difusão superficial e pelo contorno de grão são fenômenos de superfície/interface, é desejável que o aditivo esteja preferencialmente na superfície de grão. Então, uma alternativa para assegurar que o aditivo esteja realmente na superfície do material é a aplicação de um "coating" (recobrimento) do aditivo recobrindo o material a ser sinterizado. 
$\mathrm{Na}$ literatura pode-se encontrar uma grande variedade de metodologias para obtenção de recobrimento em alumina $[3,4]$. A alumina é um material cerâmico que se apresenta em diversas formas alotrópicas (estruturais) sendo a mais estável a forma $\alpha-\mathrm{Al}_{2} \mathrm{O}_{3}$, corundum. Este material é usado em diversas aplicações industriais por apresentar alta dureza e estabilidade química.

Uma rota simples para obtenção de nanocápsulas pode ser obtida pelo o uso do método sol-gel, no qual pode-se usar uma mistura de dois "sols" nanométricos, uma infiltração de um sol numa matriz porosa, microemulsão ou um gel precursor num sol nanoparticulado [5-9].

Neste trabalho será utilizado o método sol-gel, combinado com o método do precursor polimérico [10]. A grande
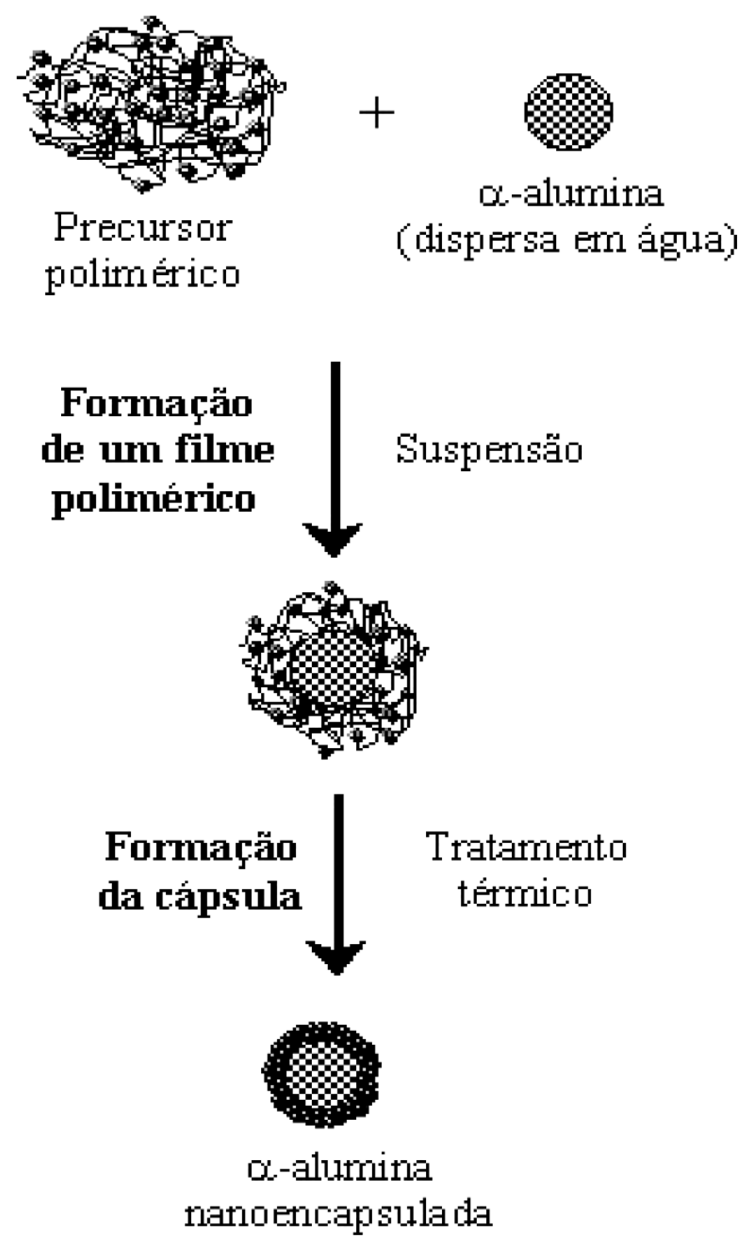

\section{- cadeia polimérica \\ - lantâtio}

Figura 1: Principais etapas para a obtenção da alumina nanoencapsulada.

[Figure 1: The main steps for obtaining nanoencapsulated alumina.] vantagem desta metodologia reside no fato de que ocorre a formação de uma fina película do precursor polimérico na superfície partículas facilitando a obtenção da nanocápsula na superfície do material em suspensão.

Este procedimento está essencialmente baseado na adição homogênea do aditivo diretamente na superfície do grão na forma de uma cápsula, depositada por método semelhante a um sol-gel modificado.

Assim, a proposta principal deste estudo está relacionada à aplicação de revestimento superficial em escala nanométrica, ou seja, o nanoencapsulamento, de $\alpha$-alumina comercial com $\mathrm{La}_{2} \mathrm{O}_{3}$, no intuito de se verificar o efeito desta nanocápsula na morfologia e microestrutura da $\alpha$-alumina.

Na Fig. 1 está ilustrada, de forma resumida, a rota de síntese para o nanoencapsulamento da $\alpha$-alumina com $\mathrm{La}_{2} \mathrm{O}_{3}$, a partir de um precursor polimérico.

\section{MATERIAIS E MÉTODOS}

O precursor, poliéster de lantânio (III), foi preparado pelo método do precursor polimérico [10]. A estrutura deste poliéster (resina) pode ser representada pela ilustração na Fig. 2. Os reagentes de partida para obtenção da resina precursora foram os seguintes: nitrato de lantânio (Mallinckrodt Baker, USA, pureza $>99,9 \%$ ), ácido cítrico (E. Merck, Alemanha, pureza $>99,9 \%$ ) e etilenoglicol (Mallinckrodt Baker, USA, pureza $>99,9 \%$ ). Foi usada $\alpha$-alumina ultrapura Tamicron TKK, Japão, (pureza > 99,99\%; tamanho médio de cristalito cerca de $25 \mathrm{~nm}$ ).

O precursor polímero de lantânio ( $1 \%$ em mol de $\mathrm{La}_{2} \mathrm{O}_{3}$ ) foi usado para encapsular a $\alpha-\mathrm{Al}_{2} \mathrm{O}_{3}$, dispersa em água destilada (10\% em massa de sólido) usando um equipamento ultrassônico (750 W, 20 kHz, Lab Trade Ltda.), à temperatura ambiente. A água foi eliminada com o auxílio de um rotaevaporador.

Os tratamentos térmicos foram realizados num forno de baixo
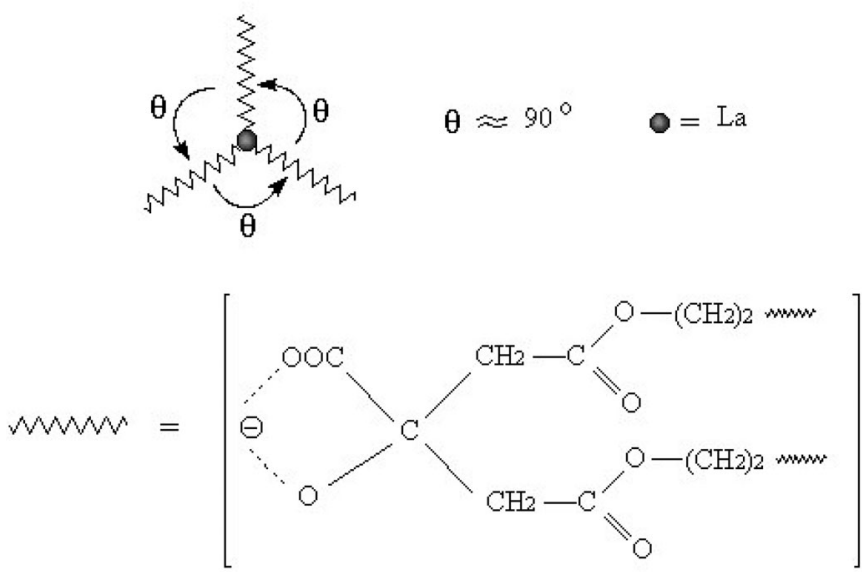

Figura 2: Estrutura do precursor polimérico de lantânio. [Figure 2: Structure of the lanthanum polymeric precursor.] 
gradiente de temperatura com um volume fixo de $50 \mathrm{~cm}^{3}$. Para as análises de difração de raios $\mathrm{X}$ foi utilizado um equipamento da Siemens, modelo D-5000, em configuração $\theta-2 \theta$.

$\mathrm{O}$ estudo de análise térmica foi realizado por meio de calorimetria diferencial exploratória - DSC (Netzsch, Pegasus 404) e dilatometria (Netzsch, DIL 402 E). As amostras de DSC foram utilizadas na forma de pó, massa $10 \mathrm{mg}$, atmosfera ar sintético, fluxo de $100 \mathrm{~cm}^{3} / \mathrm{min}$. As amostras para dilatometria foram utilizadas na forma de pastilhas ( $5 \mathrm{~mm}$ de espessura e $8 \mathrm{~mm}$ de diâmetro), prensadas isostaticamente a $210 \mathrm{MPa}$. As análises foram realizadas sob atmosfera de ar sintético, fluxo de $100 \mathrm{~cm}^{3} / \mathrm{min}$.

Para caracterização microestrutural foram obtidas micrografias de microscopia eletrônica de varredura (Zeiss DSM 940A). Para caracterização da nanocápsula em alta resolução fez-se uso da microscopia eletrônica de transmissão (Philips CM200, operando a 200 kV).

\section{RESULTADOS E DISCUSSÃO}

\section{Formação da nanocápsula de lantânio}

O procedimento utilizado facilita a obtenção da nanocápsula, uma vez que se forma inicialmente um finíssimo filme polimérico na superfície dos grãos de alumina. A Fig. 3(a) ilustra uma curva de DSC deste material. Observa-se entre 25 e $150{ }^{\circ} \mathrm{C}$ a eliminação da água e etilenoglicol residual. A partir de $200{ }^{\circ} \mathrm{C}$ tem-se início a decomposição da cadeia polimérica do precursor. Em $360{ }^{\circ} \mathrm{C}$ ocorre o máximo da degradação oxidativa do material orgânico. Por volta de $500{ }^{\circ} \mathrm{C}$ todo material orgânico foi eliminado dando origem a nanocápsula de óxido de lantânio amorfo.

A formação da nanocápsula foi confirmada com o auxílio da microscopia eletrônica de transmissão. Na Fig. 4 pode-se observar que a cápsula está homogeneamente distribuída na superfície da $\alpha-\mathrm{Al}_{2} \mathrm{O}_{3}$ com espessura variando entre 9 e $14 \mathrm{~nm}$.

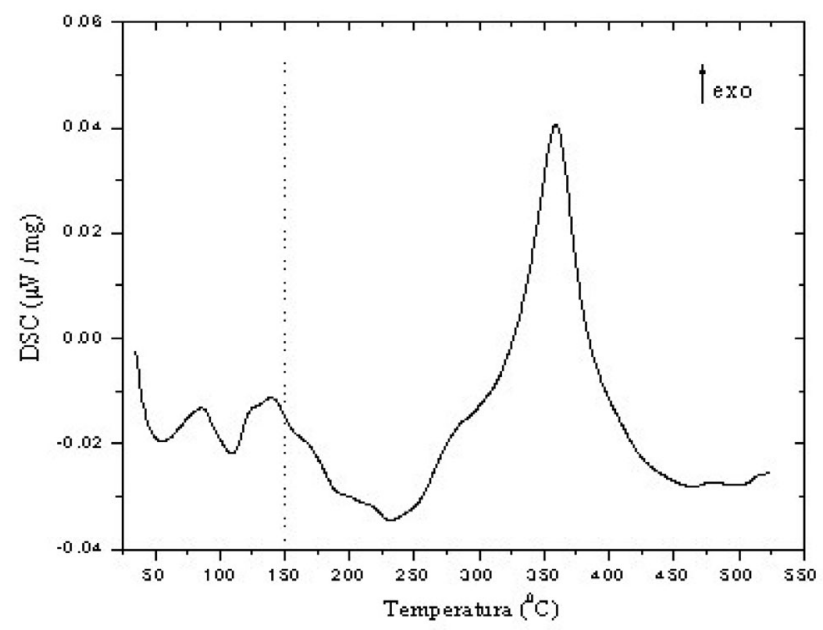

Figura 3: Curva de DSC da alumina nanoencapsulada na forma de pó. [Figure 3: DSC curve of the nanoencapsulated alumina powder.]

\section{Estudo morfológico e microestrutural dos materiais}

O máximo da retração da $\alpha$-alumina nanoencapsulada ocorre $1422{ }^{\circ} \mathrm{C}$, aproximadamente $120{ }^{\circ} \mathrm{C}$ a mais comparado com o material não encapsulado (Fig. 5). O estudo da influência da cápsula na sinterização da alumina será exposto em trabalhos futuros. Para o estudo morfológico e microestrutural serão

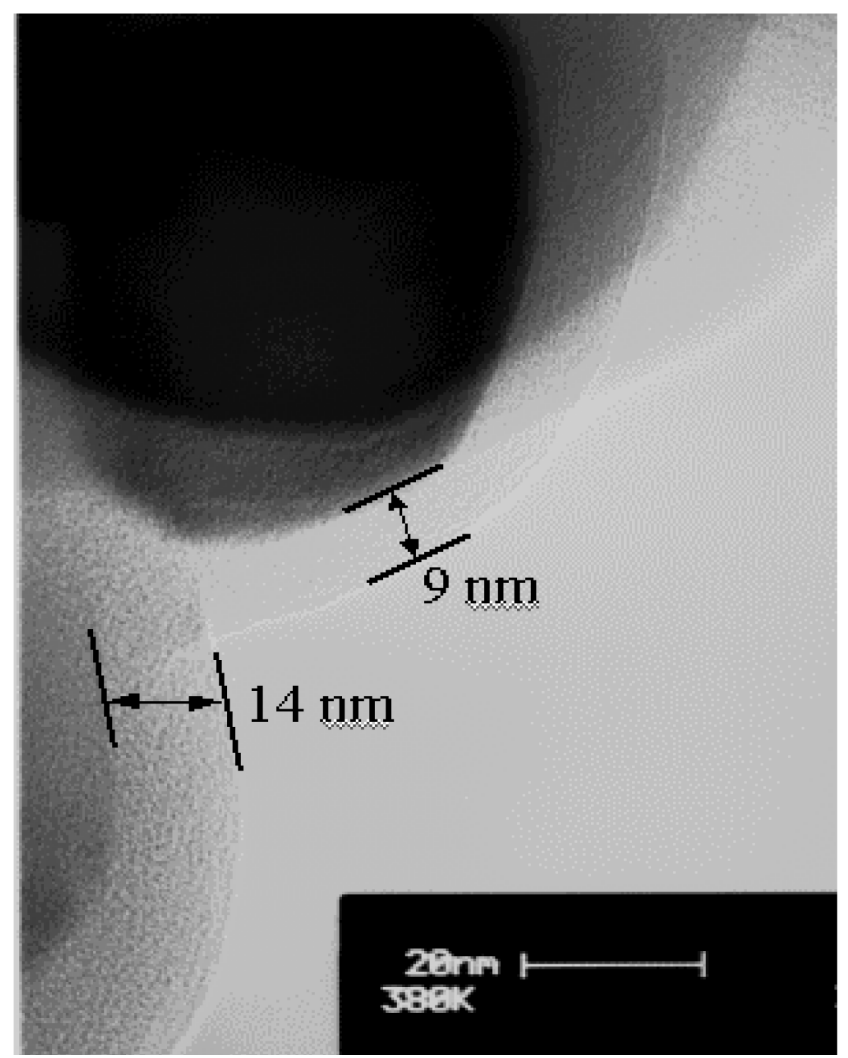

Figura 4: Imagem de microscopia eletrônica de transmissão de alta resolução da alumina nanoencapsulada.

[Figure 4: TEM high resolution micrograph of the nanoencapsulated alumina.]

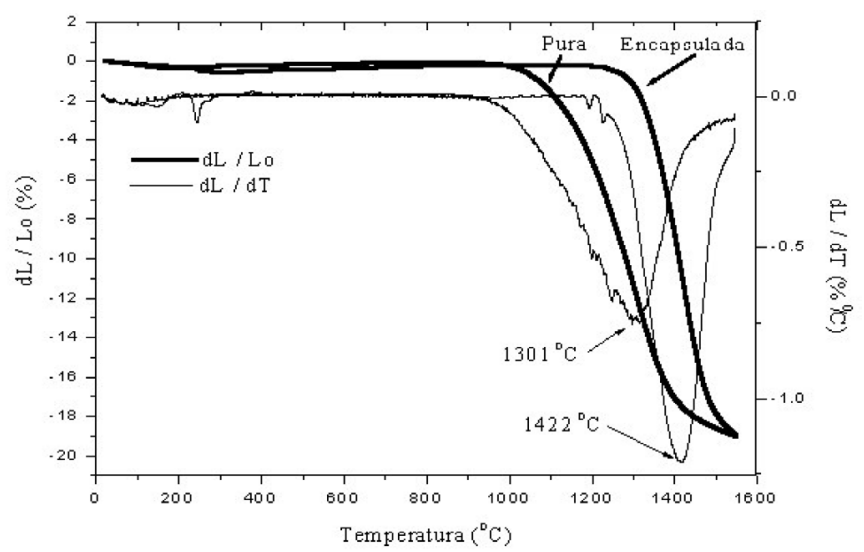

Figura 5: Curvas de dilatometria da alumina pura e nanoencapsulada.

[Figure 5: Dilatometric behaviors of pure and nanoencapsulated alumina.] 
formados dois sistemas: (A) $\alpha$-alumina nanoencapsulada na forma de pó, tratada termicamente a temperaturas até $1422^{\circ} \mathrm{C}$; e (B) $\alpha$-alumina nanoencapsulada na forma de pastilha sinterizada, tratada termicamente a $1600{ }^{\circ} \mathrm{C}$ por $2 \mathrm{~h}$.

\section{Morfologia dos grãos}

A morfologia dos grãos da alumina no sistema (A) não apresenta nenhuma modificação em relação ao material não-encapsulado, salvo, pela presença da cápsula, (Fig. 4). Então, o foco será concentrado sobre o sistema (B), no qual o material apresenta-se sinterizado.

Na Fig. 6 está ilustrada uma imagem de microscopia eletrônica de varredura da fratura de uma pastilha sinterizada de $\alpha$-alumina. Pode-se observar a presença de grãos cristalinos com diâmetro médio de $5 \mu \mathrm{m}$, com estreita distribuição de tamanho de grão, formando uma estrutura compacta.

As imagens de microscopia eletrônica de varredura da $\alpha$-alumina nanoencapsulada sinterizada (sistema B) revelaram a presença de duas regiões com características morfológicas distintas (Fig. 7). Estas regiões forma denominadas: região I, composta de grãos com tamanho médio de grão entre 1 e $2 \mu \mathrm{m}$, arredondados e alongados; região II, composta por grãos que se assemelham aos encontrados na $\alpha$-alumina não-encapsulada sinterizada. Porém, as formas desses grãos são diferentes: na $\alpha$-alumina não-encapsulada sinterizada os grãos são facetados e estão formando uma estrutura bem organizada (Fig. 6), enquanto que na região II não é observada tal regularidade.

A seguir, estas mudanças de morfologia serão discutidas em termos da microestrutura dos materiais em função do nanoencapsulamento.

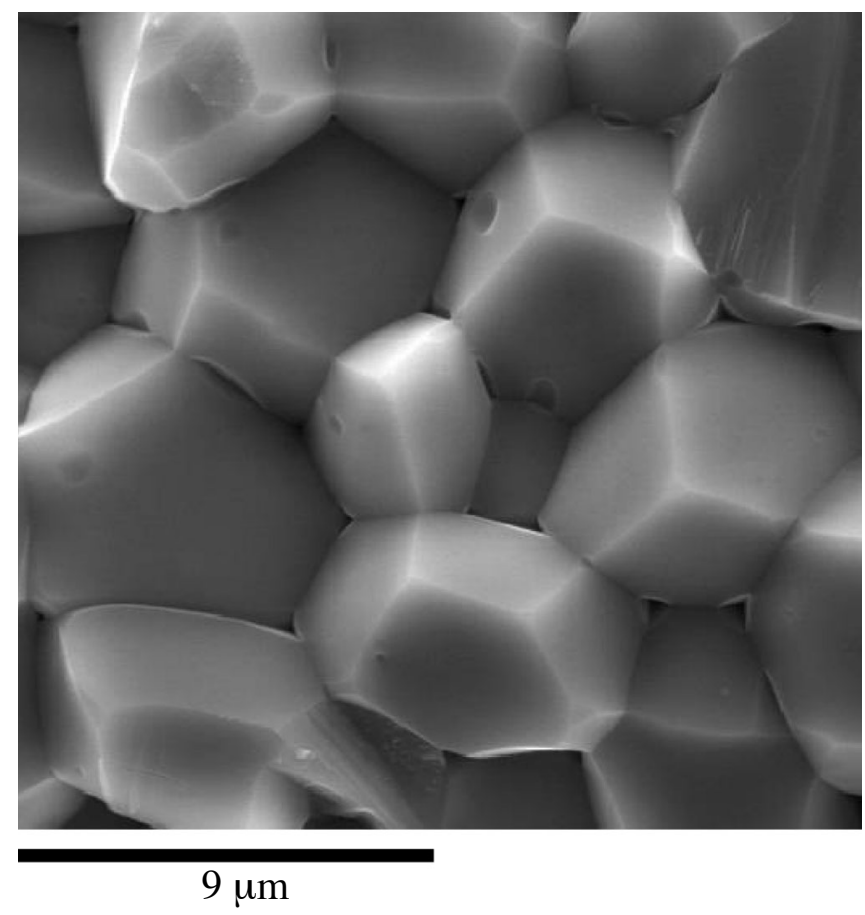

Figura 6: Imagem de microscopia eletrônica de varredura da alumina pura. [Figure 6: SEM image of pure alumina.]

\section{Microestrutura da $\alpha$-alumina nanoencapsulada}

Como exposto anteriormente, a nanocápsula de $\mathrm{La}_{2} \mathrm{O}_{3}$ começa a ser formada durante o processo de degradação da cadeia polimérica do precursor de lantânio. Assim, com a total eliminação da matéria orgânica tem-se a formação da nanocápsula amorfa, confirmada pela análise de difração de raios $\mathrm{X}$, na qual aparece apenas a fase $\alpha$ da alumina. $\mathrm{A}$ análise de difração de raios $\mathrm{X}$ do material do sistema (A), tratado a $1422{ }^{\circ} \mathrm{C}$ por $5 \mathrm{~min}$, apresentou uma segunda fase cristalina, um aluminato de lantânio simples $\left(\mathrm{LaAlO}_{3}\right)$ (Fig. $8 \mathrm{a})$, sugerindo que a cápsula não cristaliza como $\mathrm{La}_{2} \mathrm{O}_{3}$, e sim da forma $\mathrm{LaAlO}_{3}$. Com o aumento da temperatura, o lantânio dissolve-se mais na estrutura da alumina formando um aluminato complexo de fórmula $\mathrm{La}_{2} \mathrm{Al}_{24,4} \mathrm{O}_{39,6}$, que apresenta uma estrutura hexagonal semelhante à da $\alpha$-alumina. Esta fase foi observada no material sinterizado a $1600{ }^{\circ} \mathrm{C}$ por $2 \mathrm{~h}$ (sistema (B)), Fig. 8b.

A análise de EDX, Fig. 9, da região I (Fig. 7b) mostra que nesta porção do compósito o lantânio está presente, mas em pequena quantidade em relação ao alumínio. Isto sugere que o $\mathrm{La}_{2} \mathrm{Al}_{24,4} \mathrm{O}_{39,6}$ forma uma fase em separado, e não um revestimento sobre a alumina. Na região II não foi detectada a presença de lantânio pela análise de EDX.

A caracterização microestrutural da $\alpha$-alumina nanoencapsulada com lantânio servirá como suporte para estudos relacionados a compactação de cerâmicas sinterizadas com adição de aditivos superficiais, para

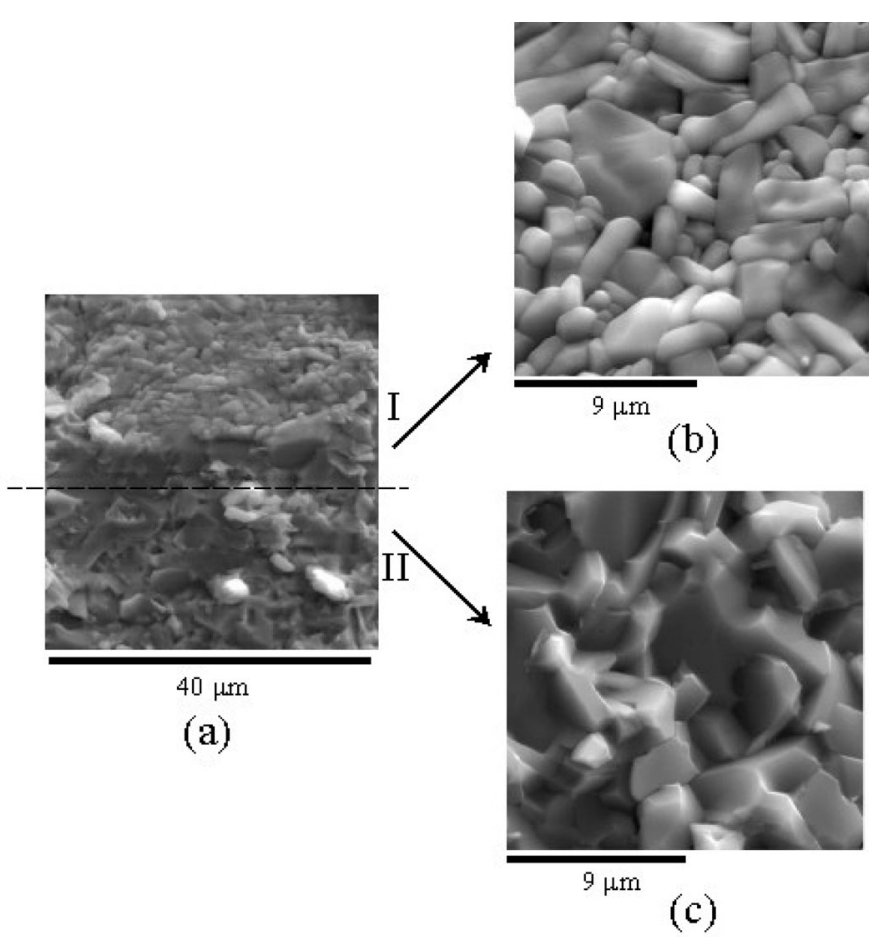

Figura 7: Imagens de microscopia eletrônica de varredura: (a) $\alpha$ alumina nanoencapsulada, (b) região I e (c) região II.

[Figure 7: SEM images: (a) nanoencapsulated $\alpha$-alumina, (b) region I e (c) region II.] 


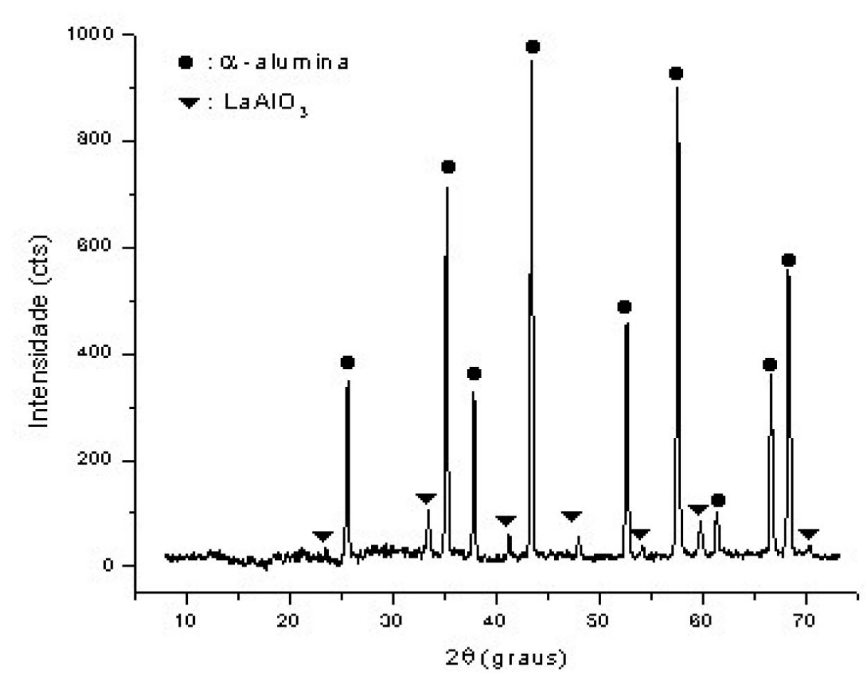

(a)

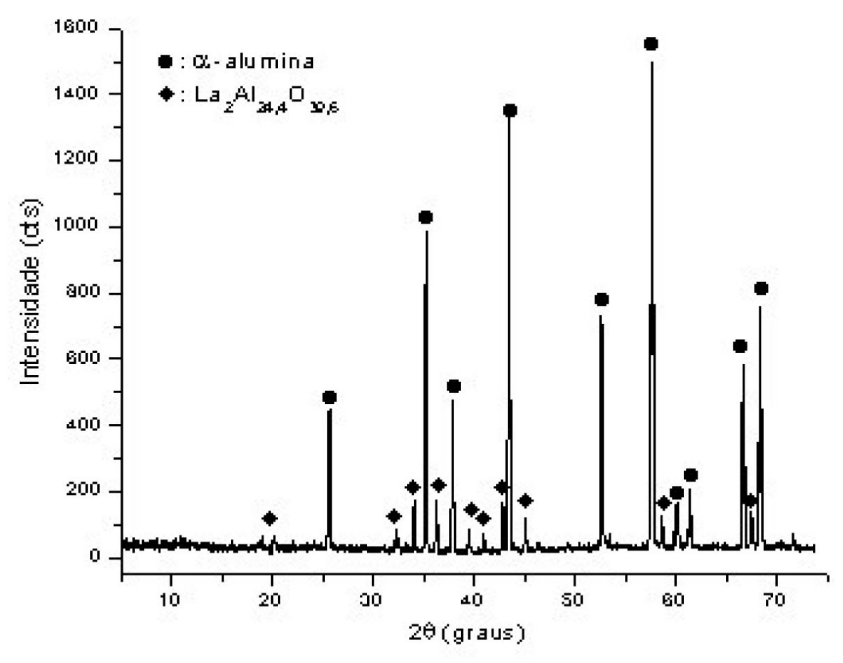

(b)

Figura 8: Difratograma de raios X da alumina nanoencapsulada: (a) antes do processo de sinterização, (b) sinterizada a1600 ${ }^{\circ} \mathrm{C}$. [Figure 8: XRD patterns of the nanoencapsulated $\alpha$-alumina: (a) before the sintering process, $(b)$ sintered at $1600^{\circ} \mathrm{C}$.]

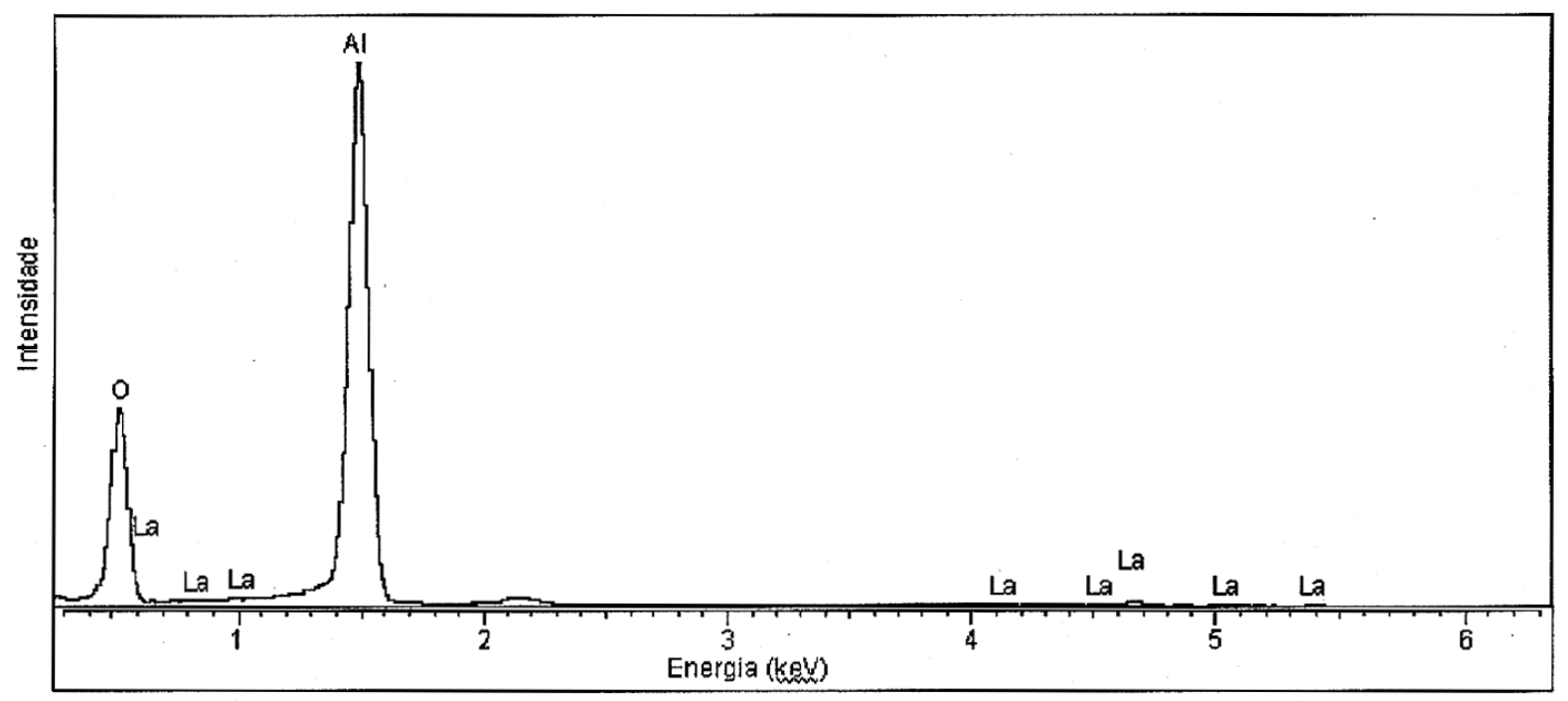

Figura 9: EDS da alumina nanoencapsulada sinterizada a $\left.1600{ }^{\circ} \mathrm{C}\right)$, região $\mathrm{I}$.

[Figure 9: EDS of the nanoencapsulated alumina sintered $\left(1600^{\circ} \mathrm{C}\right)$, region I.]

aplicações específicas. No caso da adição de terras raras à $\alpha$-alumina ocorre uma diminuição brusca do "creep" da alumina, importante propriedade para aplicação na área aeroespacial [11]; esta propriedade é altamente dependente da microestrutura do material.

\section{CONCLUSÕES}

O método sol-gel combinado ao método do precursor polimérico (sol-gel modificado) pode ser facilmente aplicado para a obtenção de nanocápsulas na superfície de óxidos cristalinos. Uma grande vantagem nesta metodologia figura-se no fato de que a formação de um finíssimo filme polimérico na superfície dos grãos facilita a obtenção de uma cápsula homogeneamente distribuída, além disso, existe uma grande facilidade em se usar o método dos precursores poliméricos para obtenção de uma grande gama de metais.

Esta metodologia será aplicada para síntese de outras nanocápsulas no intuito de se modificar a superfície de óxidos cristalinos, e conseqüentemente, conferindo a superfície da matriz, características melhoradas para aplicações específicas, tais como atividade catalítica, propriedades sensoras, dentre outras. 


\section{AGRADECIMENTOS}

Os autores agradecem o suporte financeiro das agências FAPESP, CAPES, CNPq e FINEP.

\section{REFERÊNCIAS}

[1] J. A. Varela, O. J. Whittemore, E. Longo, Ceram. Int. 16 (1990) 177.

[2] N. J. Shaw, Powder Metall. Int. 21 (1989) 31.

[3] K. Sheng-Lung, C. Yann-Cheng, G. Ming-Der, H. WenHwa, Mater. Chem. Phys. 86 (2004) 5.

[4] A. Patra, E. Sominska, S. Ramesh, Y. Koltypin, Z. Zhong, H. Minti, R. Reisfeld, A. Gedanken, J. Phys. Chem. B 103 (1999) 3361.
[5] F. Caruso, R. A. Caruso, H. Möhwald, Science 282 (1998) 1111.

[6]Y. Kobayashi, M. A. Correa-Duarte, L. M. Liz-Marzán, Langmuir 17 (2001) 6375.

[7] A. Ruys, Y. W. Mai, Mater. Sci. Eng. A 265 (1999) 202. [8] S. H. Hyun, B. S. Kang, J. Am. Ceram. Soc. 77 (1994) 3093.

[9] Y. Zhu, Y. Qian, M. Zang, Z. Chen, G. Zhou, J. Mater. Chem. 4 (1994) 1619.

[10] E. R. Leite, A. P. Maciel, I. T. Weber, P. N. LisboaFilho, E. Longo, C. O. Paiva-Santos, A. V. C. Andrade, C. A. Paskocimas, Y. Maniette, W. H. Schreiner, Adv. Mater. 14 (2002) 905.

[11] Z. Yu, G. Wu, D. Sun, J. Chen, L. Jiang, Mater. Sci. Eng. A 357 (2003) 61.

(Rec. 18/10/2004, Ac. 30/01/2005) 